\title{
BMJ Open A prospective, cross-sectional study to establish age-specific reference intervals for neonates and children in the setting of clinical biochemistry, immunology and haematology: the HAPPI Kids study protocol
}

Monsurul Hoq, ${ }^{\oplus 1,2}$ Vicky Karlaftis, ${ }^{3}$ Susan Mathews, ${ }^{4}$ Janet Burgess, ${ }^{5}$ Susan M Donath, ${ }^{1,2}$ John Carlin,, ${ }^{1,2}$ Paul Monagle, ${ }^{2,3}$ Vera Ignjatovic ${ }^{2,3}$

To cite: Hoq M, Karlaftis V, Mathews S, et al. A prospective, cross-sectional study to establish age-specific reference intervals for neonates and children in the setting of clinical biochemistry, immunology and haematology: the HAPPI Kids study protocol. BMJ Open 2019;9:e025897. doi:10.1136/ bmjopen-2018-025897

- Prepublication history and additional material for this paper are available online. To view these files, please visit the journal online (http://dx.doi. org/10.1136/bmjopen-2018025897).

Received 10 August 2018 Revised 21 February 2019 Accepted 4 March 2019

Check for updates

(C) Author(s) (or their employer(s)) 2019. Re-use permitted under CC BY-NC. No commercial re-use. See rights and permissions. Published by BMJ.

For numbered affiliations see end of article.

Correspondence to

Dr Vera Ignjatovic;

vera.ignjatovic@mcri.edu.au

\section{ABSTRACT}

Introduction The clinical interpretation of laboratory tests is reliant on reference intervals. However, the accuracy of a reference interval is dependent on the selected reference population, and in paediatrics, the ability of the reference interval to reflect changes associated with growth and age, as well as sex and ethnicity. Differences in reagent formulations, methodologies and analysers can also impact on a reference interval. To date, no direct comparison of reference intervals for common analytes using different analysers in children has been published. The Harmonising Age Pathology Parameters in Kids (HAPPI Kids) study aims to establish age-appropriate reference intervals for commonly used analytes in the routine clinical care of neonates and children, and to determine the feasibility of paediatric reference interval harmonisation by comparing age-appropriate reference intervals in different analysers for multiple analytes.

Methods and analysis The HAPPI Kids study is a prospective cross-sectional study, collecting paediatric blood samples for analysis of commonly requested biochemical, immunological and haematological tests. Venous blood samples are collected from healthy premature neonates (32-36 weeks of gestation), term neonates (from birth to a maximum of 72 hours postbirth) and children aged 30 days to $\leq 18$ years (undergoing minor day surgical procedures). Blood samples are processed according to standard laboratory procedures and, if not processed immediately, stored at $-80^{\circ} \mathrm{C}$. A minimum of 20 samples is analysed for every analyte for neonates and then each year of age until 18 years. Analytical testing is performed according to the standard operating procedures used for clinical samples. Where possible, sample aliquots from the same patients are analysed for an analyte across multiple commercially available analysers.

Ethics and dissemination The study protocol was approved by The Royal Children's Hospital, Melbourne, Ethics in Human Research Committee (34183 A). The study findings will be published in peer-reviewed journals and shared with clinicians, laboratory scientists and laboratories.
Strengths and limitations of this study

- The prospective design of the study ensures blood samples are collected from healthy neonates and children aged 30 days to $\leq 18$ years.

- Aliquots from the same blood sample are tested for common analytes on multiple commercially available analysers allowing direct head to head comparison.

- Samples are obtained from a minimum of 20 neonates and then for 20 children within each year of age until 18 years with equal numbers of males and females (total 380 samples per analyte).

- Parametric statistical methods are to be applied for estimating reference intervals that vary continuously with increasing age.

- Changes in analytes associated with age are based on samples collected cross-sectionally not based on individuals followed longitudinally.

\section{INTRODUCTION}

Reference intervals are important for the correct interpretation of clinical laboratory tests and affect the clinical assessment and care of patients. Reference intervals are used for determining normal versus abnormal for a measurement of interest, and are usually defined as a range encompassing $95 \%$ of the reference population, where lower and upper limits are 2.5th and 97.5th centile, respectively. ${ }^{1}$

Obtaining accurate data from a suitable reference population is an essential element in determining reference intervals, and therefore the characteristics of a reference population need to be matched to a 'normal' or a healthy population using strict criteria. However, collecting blood samples from 
children for different laboratory tests is challenging due to the small blood volume that can be safely collected from children and the ethical concern of obtaining blood from healthy children. ${ }^{2}$ For these reasons, previously documented paediatric reference interval studies are frequently based on retrospective analysis of laboratory data, prospective sample collection from school children in the community (ie, limited age groups) or from paediatric hospitals where children are undergoing minor elective surgery. ${ }^{3-7}$ Regardless of how the samples are collected, very few of these studies have included neonates through to 18 years of age. ${ }^{8}$

In children, development and growth are among the key challenges in determining reference intervals for different analytes. ${ }^{2}$ The dynamic nature of different analytes as children grow and develop influences the representation of paediatric reference intervals, which have often historically been established or partitioned for different age ranges. ${ }^{89}$ This approach can often lead to misdiagnosis, especially when the age of the child that is being investigated is in close proximity to the borders of the partitions. For example, a child aged 3 years and 360 days may have clinical results that would be classified as outside the reference interval of $0-4$ years for a blood test of interest, but within the reference interval for children aged 4-9 years. Hence, the difference of 5 days results in the test result being labelled as abnormal. Misdiagnosis leads to repeated blood tests for the child, increased utilisation of health services and is often associated with anxiety for parents and children alike. The determination of the age-specific partition points has often been arbitrary and often there is not an even distribution of the number of reference subjects included across different partitions. For these and other reasons, the process of determining age-specific reference intervals for different analytes based on the artificial partitioning of age has been found to be problematic. ${ }^{4} \mathrm{~A}$ preferred approach, which addresses the age-related changes in analyte levels, is to construct age-appropriate reference intervals considering age as a continuous variable. ${ }^{1410}$

The laboratory test results for most analytes are dependent on the analyser and reagents used for analysis and therefore, analyser-specific and reagent-specific reference intervals should be determined. In several studies, mathematical algorithms or 'transference' mechanisms have been applied to compare and construct reference intervals for different analysers based on the reference values for one specific analyser. ${ }^{11}{ }^{12}$ However, to date, direct comparison of the reference intervals for common analytes using different analysers has not been undertaken in neonates and children.

The Harmonising Age Pathology Parameters in Kids (HAPPI Kids) study is designed to address the existing gaps in reference interval studies with prospectively collected blood samples from healthy neonates and children, from birth until 18 years of age.

\section{METHODS AND ANALYSIS}

\section{The HAPPI Kids study: aims}

- Establish age-appropriate reference intervals for commonly used analytes in clinical practice in the setting of neonates and children.

- Determine whether analyser characteristics alter age-appropriate reference intervals for multiple analytes, in order to determine whether paediatric reference intervals can be harmonised.

\section{Study design}

The HAPPI Kids study is a prospective cross-sectional study for the collection of paediatric blood samples for commonly requested biochemical, immunological and haematological analysis.

\section{Study subjects}

The study subjects consist of healthy premature neonates (32-36 weeks of gestation), term neonates (from birth up to a maximum of 72 hours postbirth) and children aged 30 days to 18 years (undergoing minor day surgical procedures).

\section{Participant recruitment and sample collection}

Participant recruitment commenced in February 2015 in four major public hospitals in Melbourne, Australia. Parents or guardians of eligible study participants are approached by pathology collection staff at each site and written consent is obtained following a verbal and written explanation of the study and participation requirements. Following patient consent, the pathology collection staff interview the parents or guardian using the study questionnaire (online supplementary table 1) to confirm study inclusion and exclusion criteria status (table 1). In addition, the child's medical record is reviewed to assess and document the child's general health.

Three distinct routes of patient recruitment are utilised: 1. Preterm neonatal samples are collected from 'healthy' neonates about to be discharged from postnatal wards, born at 32-36 weeks of gestation, at The Royal Women's Hospital. Samples are collected in the first 3 days of life via direct venepuncture, using a 23-gauge needle, into required blood collection tubes. The preterm neonates do not have any systemic abnormalities, that is, underlying diseases and comorbidilites, abnormal foetal monitoring and do not require any mechanical ventilation.

2. Term neonatal samples are collected from healthy term neonates about to be discharged from postnatal wards following routine intramuscular administration of $1 \mathrm{mg}$ of vitamin $\mathrm{K}$ in the delivery suites at The Royal Women's Hospital, Northern Health and Western Health-Sunshine Hospital. Samples are collected from birth up to a maximum of 72 hours postbirth in hospital via direct venepuncture, using a 23-gauge needle into the required blood collection tubes (Sarstedt). To minimise the number of blood collection procedures performed, the venous sample is also utilised for 
Table 1 Summary of the inclusion and exclusion criteria at screening

\begin{tabular}{lll}
\hline & Inclusion criteria & Exclusion criteria (any one) \\
\hline Age groups & Gestational age of $32-36$ weeks. \\
Premature neonates & Generally healthy. \\
Term neonates & From bequires interpreter. \\
& Gestational age to $\geq 37$ weeks \\
& APGAR score $\geq 7$ at 5 min. \\
Paediatric & Attending hospital for minor elective surgery requiring \\
& general anaesthetic $(30$ days to 18 years) or volunteer \\
& to participate in the study after seeing a flyer related to \\
& the study (15-18 years).
\end{tabular}

\section{Category}

Haematology

Immunology
Presence of coagulation disorders.

Family history of coagulation disorders.

Currently on anticoagulation medication.

- Presence of immune system disorder or immune deficiency syndrome.

- Presence of genetic disorder.

- Presence of rheumatological disorder.

- Family history of rheumatological disorder or immune deficiency syndrome

- Infection or a febrile illness within the last 7 days.

- Infection or a febrile illness within the last 4 weeks.

- Hospital admission for intravenous antibiotics to clear an infection on more than two occasions in life.

- Has needed 2 or more months of oral antibiotics more than two occasions in their life.

- Failure to thrive.

- Recipient of blood products in the last 3 months.

- Diagnosed with food allergy, asthma, eczema or hayfever.

- Family history of food allergy, asthma, eczema or food allergy.

Biochemistry

- History of liver and renal disease.

Presence of endocrine diseases.

- Presence of metabolic disease.

- Presence of renal disease.

- Presence of hepatic disease.

Failure to thrive.

APGAR, appearance, pulse, grimace, activity, rerspiration; ID, identification.

application to the routine newborn screening Guthrie card.

3. Blood samples from healthy children aged 30 days to 18 years are collected at The Royal Children's Hospital, Northern Hospital and Western HealthSunshine Hospital. The samples are obtained from healthy children prior to minor elective day surgery (eg, circumcision). Other than elective surgery, these children are deemed healthy and are not receiving any medications. All children are fasted before their elective surgery.

Only one attempt to collect blood per participant, per sample is made during the HAPPI Kids study. Blood samples are collected into specific tubes required by the analyser manufacturer depending on the analytes being tested (eg, serum vs citrate). The participant recruitment and sample collection are currently ongoing.

\section{Sample size}

A minimum of 20 samples is collected for 19 specific age groups, for exapmle, neonates and every year of life from 30 days to 18 years. ${ }^{1}$ For the biochemical and haematological parameters, a total of 380 samples are analysed. For the immunological analysis, the number of age groups is increased from 19 to 22 (ie, 1-2 months, 2-6 months, 6-9 months, 9-12 months, 12-18 months, 18-24 months and every year of life from 3 to 18 years), equating to a total of 440 samples. For preterm neonates, a total of 100 samples are collected to facilitate analysis of 20 samples for each week of gestation from 32 to 36 weeks.

The sample size of 380 or 440 for estimating $95 \%$ reference intervals of an analyte using parametric methods with samples distributed uniformly across 30 days to $<18$ years is considered adequate based on the approach proposed by Royston. ${ }^{1}$ Using a formula for the approximate SE of 
the reference limit at the mean value of age, he suggested 292 as a suitable sample size for constructing a $95 \%$ reference intervals based on restricting the SE of the limits of the resulting reference intervals to be no $>10 \%$ of the residual SD from the parametric model. ${ }^{1}$

\section{Sample processing and storage}

Samples are thawed as per standard clinical laboratory practice for the analyte in question. There are no repeat freeze-thaw cycles for any of the samples. For some analytes, the samples are processed immediately and not frozen, within the same time frame and conditions under which clinical samples would be handled. Analytes that can be frozen will be tested in batches. Testing will be done with samples from all ages included in each batch with randomly assigned orders of testing to avoid any batch to batch bias.

For biochemistry analytes, samples are collected into S-Monovette serum gel tubes (Sarstedt), centrifuged at $5000 \mathrm{rpm}$ and $6^{\circ} \mathrm{C}$ for $5 \mathrm{~min}$, separated and stored in $400 \mu \mathrm{L}$ aliquots at $-80^{\circ} \mathrm{C}$ within 4 hours of collection.

For immunology analytes, samples are collected into either S-Monovette serum gel tubes (Sarstedt) or S-Monovette neutral tubes (Sarstedt), depending on the downstream tests. The samples are spun at $5000 \mathrm{rpm}$ and $6^{\circ} \mathrm{C}$ for $5 \mathrm{~min}$, separated and the resultant serum stored in $400 \mu \mathrm{L}$ aliquots at $-80^{\circ} \mathrm{C}$ within 4 hours of collection.

For haematology analytes, samples are collected into S-Monovette citrate $3.2 \%$ tubes (Sarstedt). The samples are centrifuged at $3800 \mathrm{rpm}$ at room temperature for $5 \mathrm{~min}$, and plasma is stored in $500 \mu \mathrm{L}$ aliquots at $-80^{\circ} \mathrm{C}$ within 4 hours of collection, using a previously established collection protocol. ${ }^{13}$

Samples utilised for determination of blood groups $(\mathrm{ABO})$ and the thalassemia screen are collected into $500 \mu \mathrm{L}$ S-Monovette EDTA tubes and processed in the laboratory within 24 hours of collection.

Cell count samples are collected in S-Monovette EDTA tubes (Sarstedt) and processed by the laboratory within 3 hours of collection.
The details of blood tubes used in sample collection are provided in table 2 .

\section{Sample testing}

Blood samples collected from the study participants are tested according to documented and accredited standard operating procedures used for the testing of patient samples within the participating laboratories. The biochemical, immunological and haematological analytes tested are listed in table 3. Where indicated (*), sample aliquots from the same patient are tested for the same analyte using different automated analysers commonly in clinical use to facilitate direct head-to-head comparison. In addition, where indicated in table $3(\dagger)$, aliquots of the same sample are tested in different laboratories using the same analyser type and test method. The sample testing is currently ongoing.

\section{Data management system}

Study data are collected and managed using the Research Electronic Data Capture (REDCap) electronic data capture system hosted at the Murdoch Children's Research Institute. ${ }^{14}$ REDCap is a secure, web-based application designed to support data capture for research studies, providing (1) an intuitive interface for validated data entry, (2) audit trails for tracking data manipulation and export procedures, (3) automated export procedures for seamless data downloads to common statistical packages and (4) procedures for importing data from external sources.

\section{Data analysis}

Test results for different analytes will be plotted according to age in order to identify outliers, explore normality of distributions and to assess the association between analyte level and age. Outliers will to be excluded from analysis following a detailed examination, including visual inspection, appropriate statistical tests, for example, Tukey's test and assessment of biological implausibility, for example, haemolysis index for biochemistry samples. Reference intervals will be constructed using parametric statistical

\section{Table 2 Blood tubes used in sample collection}

\begin{tabular}{lllllll}
\hline $\begin{array}{l}\text { Analytes } \\
\text { category }\end{array}$ & Tube type & Manufacturer & Subtype & Volume $(\mathbf{m L})$ & Product code & Age group \\
\hline Biochemistry & SST & Sarstedt & S-Monovette & 7.5 & 01.1602 .001 & Neonate and paediatric \\
Haematology & EDTA & Sarstedt & S-Monovette & 2.7 & 05.1167 .001 & Paediatric \\
& EDTA & Sarstedt & Micro Tube & 0.5 & 41.1395 .002 & Neonate \\
& Sodium citrate & Sarstedt & S-Monovette & 3 & 05.1165 .100 & Paediatric \\
& Sodium citrate & Sarstedt & S-Monovette & 1.4 & 06.1668 .100 & Neonate \\
& Lithium heparin & Sarstedt & S-Monovette & 7.5 & 01.1608 .001 & Paediatric \\
& Lithium heparin & Sarstedt & & 0.5 & 20.1345 & Neonate \\
& SST & Sarstedt & S-Monovette & 7.5 & 01.1602 .001 & Neonate and paediatric \\
& Neutral & Sarstedt & S-Monovette & 7.5 & 01.1728 .001 & Neonate and paediatric \\
\hline
\end{tabular}

SST, serum separator tube. 
Table 3 List of analytes tested for the HAPPI Kids study

\begin{tabular}{|c|c|c|}
\hline Biochemical & Immunological & Haematological \\
\hline Sodium*†‡ & $\lg G^{*}$ & Factors: XII, XI, IX, X, II, VII, VIII, V \\
\hline Potassium* ${ }^{*} \ddagger$ & $\lg A^{*}$ & $\begin{array}{l}\text { Inhibitors: protein C, protein S, antithrombin, } \\
\text { alpha-2-macroglobulin }\end{array}$ \\
\hline Chloride*†‡ & $\lg M^{*}$ & Von Willebrand factor \\
\hline Bicarbonate* $† \ddagger$ & Rheumatoid factor* & Collagen binding assay \\
\hline Urea*†‡ & Complement component $3^{*}$ & Ristocetin cofactor assay \\
\hline Creatinine* $† \ddagger$ & Complement component $4^{*}$ & Activated partial thromboplastin time $\neq$ \\
\hline Total bilirubin $\ddagger$ & Cystatin $\mathrm{C}^{*}$ & Prothrombin time $\ddagger$ \\
\hline Conjugated bilirubin ${ }^{*} † \ddagger$ & Anti-streptolysin O test* & Fibrinogen $\ddagger$ \\
\hline Alkaline phosphate* $† \ddagger$ & Thyroid peroxidase* & Thrombin clotting time \\
\hline Aspartate aminotransferase * $† \ddagger$ & Thyroglobulin* & D-dimers $\ddagger$ \\
\hline Alanine aminotransferase * $† \ddagger$ & Iron* & Full blood examination and reticulocytes \\
\hline Gamma-glutamyl transferase * $† \ddagger$ & Ferritin* $\ddagger$ & Red cell folate \\
\hline Total protein ${ }^{\star} \dagger$ & Transferrin* & Glucose-6-phosphate dehydrogenase \\
\hline Albumin ${ }^{*} \dagger$ & Antinuclear antibody & Active B12 \\
\hline Calcium†‡ & Soluble FAS ligand & Total homocysteine \\
\hline Magnesium${ }^{\star} \dagger \ddagger$ & Soluble CD25 & Total B12 \\
\hline Phosphate $^{\star} \dagger \ddagger$ & $\lg E$ & Serum folate \\
\hline Lactate dehydrogenase ${ }^{*} \dagger$ & Classic haemolytic complement pathway & \\
\hline Creatine kinase ${ }^{*} \dagger$ & Alternative haemolytic complement pathway & \\
\hline Lipase* $†$ & $\begin{array}{l}\text { Mannose-binding lectin complement } \\
\text { pathway }\end{array}$ & \\
\hline
\end{tabular}

\section{Amylase* $\dagger$}

Uric acid $^{*} \dagger$

Triglycerides * $\dagger$

Cholesterol $^{*} \dagger$

High-density lipoprotein ${ }^{\star} \dagger$

Thyroid-stimulating hormone ${ }^{*} \dagger$

Free thyroxine ${ }^{*} \dagger$

Free triiodothyronine ${ }^{*} \dagger$

Anti-Müllerian hormone*

Oestradiol*

Sex hormone-binding globulin*

Dehydroepiandrosterone-sulfate*

Cortisol $^{\star}$

Growth hormone*

Testosterone*

25-Hydroxyvitamin $D^{*}$

High-sensitivity oestradiol*

Androstenedione*

$17 \alpha$-Hydroxyprogesterone*

Insulin-like growth factor $1^{*}$

Insulin-like growth factor-binding protein-3*

*Sample aliquots from the same patient are tested for the same analyte using different automated analysers in common clinical use to facilitate direct head-to-head comparison.

†Aliquots of the same sample are tested in different laboratories using the same analyser type and test method.

‡Samples from preterm children are tested.

HAPPI Kids, Harmonising Age Pathology Parameters in Kids. 
methods in two steps. In the first step, a fractional polynomial regression model of age will be fitted to the mean of normally distributed or $\log$ transformed test results of an analyte, using sex as a covariate. ${ }^{15}$ In the second step, the 2.5th and 97.5th centiles will be estimated using quantile regression where the power variables of age from the fractional polynomial regression model are used as covariates. ${ }^{16}$ Potential interaction with sex will be examined. The $95 \%$ CI of the reference intervals will be estimated based on the fitted model for the reference limits. A combination of statistical testing, that is, goodness of fit and variance component analysis, and clinical expertise will be used to determine the extent to which the reference intervals of an analyte based on different analysers can be compared.

Stata V.15 will be used for data analysis. ${ }^{17}$

\section{Study timeframe}

The participant recruitment and sample collection commenced on February 2015 and is expected to be completed by August 2021. The testing of samples for 30 biochemical analytes has commenced, while testing of samples for the remaining biochemical analytes and immunological and haematological analytes are currently ongoing. We plan to complete the testing of sample by February 2021.

\section{Patient and public involvement}

The study was conceived in response to patient complaints about having blood tests done at community pathology laboratories and their children requiring repeat testing because age-appropriate normal data were not available. The recruitment and consent processes were piloted and parental/child feedback incorporated into the main study protocol. A summary of the results will be shared by mail with all the participants involved. If any child has a clinically abnormal result, the parents are contacted and appropriate plans for review of the child are made.

\section{ETHICS AND DISSEMINATION}

The study is primarily supported by The Royal Children's Hospital Foundation and is based at the Haematology Research Laboratory, Murdoch Children's Research Institute, Melbourne, Australia.

Written consent is obtained from parents or guardians of eligible study participants following a verbal and written explanation of the study and participation requirements by Pathology Collection staff at each site after. Less than $3 \%$ of the blood volume is collected from a child considering the amount of blood volume a child has, while only one attempt is made to minimise risk associated with collecting intravenous blood.

Participants' identifying information is replaced by a study number (ID) at recruitment to maintain confidentiality. This ID is used in all laboratory specimens, evaluation forms, reports and other records that leaves the site. Electronic data are stored in REDCap which can only be accessed by the authorised members of the research team. Similarly, paper-based information are stored in a locked filing cabinet and can only be accessed by the designated persons. The blood samples are stored in freezers that are only accessible to authorised members of the research team by using their individual swipe card maintaining a record of who has accessed the samples and at what time.

All results that fall outside the existing reference range used by the clinical laboratory are referred immediately to the study coordinator to determine whether there is any clinical significance that requires follow-up for the child. If necessary, families are contacted and the blood tests repeated, or an appropriate referral will be made and the individual will be followed up by the appropriate specialist.

At the conclusion of the study, blood samples collected as part of this study will be destroyed, unless consent has been given for them to be stored in a biobank located at the Haematology Research Laboratory for use in future research.

The study results will be summarised for submission to peer-reviewed journals. Results will be shared with participating hospitals and laboratories and presented at local meetings, national and international conferences.

\section{DISCUSSION}

Reference intervals are used on a daily basis by clinicians to interpret measurements obtained from patients. ${ }^{18}$ The accuracy and reliability of the reference intervals are highly dependent on the reference population utilised to define those reference intervals. ${ }^{11} 19$ As recommended by Eugeneand James, the characteristics of the sample from the reference population should be similar to those of the patients. ${ }^{20}$ Paediatric reference intervals established by laboratories using retrospective data mining techniques provide extensive sample numbers. However, the representativeness of the population is often compromised by using the laboratory results of children who were initially referred for a significant clinical investigation. ${ }^{8}$ Samples collected prospectively from preschools are useful in constructing reference intervals, but are applicable to preschool aged children only. ${ }^{6}$ Addressing this issue, the Canadian Initiative in Paediatric Reference Intervals (CALIPER) and German Health Interview and Examination Survey for Children and Diagnosis collected data prospectively using a community-based approach ensuring representativeness. ${ }^{10} 2122$ Similarly, the HAPPI Kids study is one of the very few studies collecting data prospectively from predefined healthy preterm neonates and term neonates from birth until 72 hours postbirth, as well as children from 30 days to 18 years of age.

Samples collected for HAPPI Kids use paediatric pathology collectors with specific skills and documented competency to collect venous samples from neonates. All collected samples meet Royal Children's Hospital (RCH) laboratory standard operating procedures for collection and storage as documented in the specimen collection 
handbook for the analytes. ${ }^{23}$ All participating laboratories perform daily quality control, participated in external quality assurance programmes (Royal College of Pathologists of Australasia Quality Assurance Programs) and were accredited by the National Association of Testing Authorities. The prospective design of the HAPPI Kids study also ensures quality of sample collection, storage and testing in different laboratories.

The Clinical \& Laboratory Standards Institute (CLSI) recommended sample size for establishing reference intervals for a discrete age group is $120 .{ }^{12}$ However, there is no recommendation for sample size when reference intervals are constructed for continuous age. Few papers have previously reported continuous reference intervals across childhood and are based on retrospective data mining of samples taken for clinical purposes over many years. ${ }^{424}$ No previous studies have collected prospective samples (which were not required for clinical management) from neonates and children specifically screened to be a 'well population' for the purpose of developing continuous reference intervals. In that context, the exact numbers required remain unknown. We judged that a similar degree of precision in estimation of the limits of agreement to that proposed by Royston, ${ }^{1}$ that is, requiring the ratio of the SE of the estimated limits to be no $>10 \%$ of the SD of the variation in the population, would be appropriate in our setting. Planning to recruit 380 children assured us of meeting this criterion. ${ }^{1}$ However, it is our intention to do a simulation study to develop better guidance for appropriate numbers of samples required to allow reliable characterisation of age-specific analyte dynamics.

The direct comparison of reference intervals by analysers is not possible using data mining techniques, nor when testing remainder of clinically driven samples (due to sample aliquot limitations). To overcome this problem, CALIPER programwhich initially established reference intervals using the Abbott ARCHITECT analyser transferred reference intervals in line with CLSI recommendations by testing only 100 samples on Beckman Coulter DxC800, Ortho Vitros 5600, Roche Cobas 600 and Siemens Vista 1500 using an algorithm. ${ }^{11}{ }^{12}$ The study used $\mathrm{r}^{2}<0.70$ as an indicator of non-transference between Abbott ARCHITECT and another platform and reported several analytes as non-transferable between analysers. ${ }^{11}$ The challenges in transference from the Abbott ARCHITECT to another analyser were discussed by the authors including the lack of an appropriate number of samples per age group since only 100 samples were tested to predict the linear relationship. ${ }^{11}$ The HAPPI Kids study utilises aliquots of the same blood sample from the same patient and tests the aliquots on different analysers, enabling the first documented head-to-head comparison between analysers for the age spectrum covering neonates to children 18 years of age. Therefore, we expect the results of the HAPPI Kids study to be very useful in improving interpretation of pathology results for neonates and children.

\section{Author affiliations}

${ }^{1}$ Department of Clinical Epidemiology and Biostatistics Unit, Murdoch Children's Research Institute, Parkville, Victoria, Australia

${ }^{2}$ Department of Paediatrics, The University of Melbourne, Parkville, Victoria, Australia ${ }^{3}$ Department of Haematology Research, Murdoch Children's Research Institute, Parkville, Victoria, Australia

${ }^{4}$ Department of Biochemistry, Laboratory Services, The Royal Children's Hospital, Parkville, Victoria, Australia

${ }^{5}$ Department of Pathology Collection, Laboratory Services, The Royal Children's Hospital, Parkville, Victoria, Australia

Contributors $\mathrm{MH}$ wrote the manuscript and developed the statistical analysis plan. VK wrote the study protocol, obtained ethics approval for the study, cowrote the manuscript and is the coordinator of the study. SM provided biochemistry expertise in the design of the study and revised the manuscript. JB provided pathology collection expertise in the design of the study, training for the pathology collectors and reviewed the manuscript. JC and SMD provided support for statistical analysis plan and reviewed the manuscript. PM conceived the study and contributed to its design and was a major contributor in writing the manuscript. VI contributed to the design of the study and was a major contributor in writing the manuscript. All authors read and approved the final manuscript.

Funding This study has been primarily funded by The Royal Children's Hospital Foundation (Proposal ID: 2014 - 233), with supplementary funding from Ortho Diagnostics. In addition, the study received in-kind reagents from Roche Diagnostics. Funding bodies do not have any input in the design of the study, sample collection, analysis and interpretation of data and in preparation of the manuscript.

Competing interests None declared.

Patient consent for publication Parental/guardian consent obtained.

Ethics approval The study protocol has been approved (34183 A) by The Royal Children's Hospital, Melbourne, Ethics in Human Research Committee (HREC) and subsequently approved by the HREC committees of all participating hospitals including The Royal Women's Hospital, Northern Health and Western HealthSunshine Hospital.

Provenance and peer review Not commissioned; externally peer reviewed.

Open access This is an open access article distributed in accordance with the Creative Commons Attribution Non Commercial (CC BY-NC 4.0) license, which permits others to distribute, remix, adapt, build upon this work non-commercially, and license their derivative works on different terms, provided the original work is properly cited, appropriate credit is given, any changes made indicated, and the use is non-commercial. See: http://creativecommons.org/licenses/by-nc/4.0/.

\section{REFERENCES}

1. Royston P. Constructing time-specific reference ranges. Stat Med 1991;10:675-90.

2. Coffin CM, Hamilton MS, Pysher TJ, et al. Pediatric laboratory medicine: current challenges and future opportunities. Am J Clin Pathol 2002;117:683-90.

3. Adeli K. Closing the gaps in pediatric reference intervals: the CALIPER initiative. Clin Biochem 2011;44:480-2.

4. Loh TP, Antoniou G, Baghurst P, et al. Development of paediatric biochemistry centile charts as a complement to laboratory reference intervals. Pathology 2014;46:336-43.

5. Pediatric reference intervals. Pediatric reference intervals: critical gap analysis and establishment of a national initiative. Clin Biochem 2006;39:559-60.

6. Southcott EK, Kerrigan JL, Potter JM, et al. Establishment of pediatric reference intervals on a large cohort of healthy children. Clin Chim Acta 2010;411:1421-7.

7. Shaw JL, Cohen A, Konforte D, et al. Validity of establishing pediatric reference intervals based on hospital patient data: a comparison of the modified Hoffmann approach to CALIPER reference intervals obtained in healthy children. Clin Biochem 2014;47:166-72.

8. Colantonio DA, Kyriakopoulou L, Chan MK, et al. Closing the gaps in pediatric laboratory reference intervals: a CALIPER database of 40 biochemical markers in a healthy and multiethnic population of children. Clin Chem 2012;58:854-68.

9. Daly CH, Liu X, Grey VL, et al. A systematic review of statistical methods used in constructing pediatric reference intervals. Clin Biochem 2013;46:1220-7. 
10. Kohse KP. KiGGS - the German survey on children's health as data base for reference intervals and beyond. Clin Biochem 2014;47:742-3.

11. Estey MP, Cohen AH, Colantonio DA, et al. CLSI-based transference of the CALIPER database of pediatric reference intervals from abbott to beckman, ortho, roche and siemens clinical chemistry assays: direct validation using reference samples from the CALIPER cohort. Clin Biochem 2013;46:1197-219.

12. CLSI. CLSI Releases guidelines for defining, establishing, and verifying reference intervals in the clinical laboratory: COMTEX News Network, Inc, 2008.

13. Monagle $\mathrm{P}$, Barnes $\mathrm{C}$, Ignjatovic $\mathrm{V}$, et al. Developmental haemostasis. Impact for clinical haemostasis laboratories. Thromb Haemost 2006;95:362-72.

14. Harris PA, Taylor R, Thielke R, et al. Research electronic data capture (REDCap)--a metadata-driven methodology and workflow process for providing translational research informatics support. J Biomed Inform 2009;42:377-81.

15. Royston P, Wright EM. A method for estimating age-specific reference intervals ('normal ranges') based on fractional polynomials and exponential transformation. . Series A (Statistics in Society): Journal of the Royal Statistical Society, 1998:161. 79-101.
16. Wei Y, Pere A, Koenker R, et al. Quantile regression methods for reference growth charts. Stat Med 2006;25:1369-82.

17. StataCrop. Stata statisical software: release 15. Colleage Station, TX: Stata Crop LP, 2017.

18. Koerbin G, Tate JR. Harmonising adult reference intervals in Australia and New Zealand - the continuing story. Clin Biochem Rev 2016;37:121-9.

19. Ritchie RF, Palomaki G. Selecting clinically relevant populations for reference intervals. Clin Chem Lab Med 2004;42:702-9.

20. Eugene $\mathrm{KH}$, James CB. Statistical bases of reference values in laboratory medicine. New York : Marcel Dekker, Inc, 1995.

21. Adeli K, Higgins V, Trajcevski K, et al. The Canadian laboratory initiative on pediatric reference intervals: a CALIPER white paper. Crit Rev Clin Lab Sci 2017;54:358-413.

22. Ridefelt $P$. Pediatric reference intervals - the Swedish experience. Clin Biochem 2014;47:740-1.

23. Melbourne TRCsH. RCH Specimen collection handbook. https:// ww2.rch.org.au/specimen

24. Zierk J, Arzideh F, Rechenauer T, et al. Age- and sex-specific dynamics in 22 hematologic and biochemical analytes from birth to adolescence. Clin Chem 2015;61:964-73. 